\title{
CITRIC: cold-inducible translational readthrough in the chloroplast of Chlamydomonas reinhardtii using a novel temperature-sensitive transfer RNA
}

Rosanna Young ${ }^{1,2}$ a and Saul Purton ${ }^{1 *}$ (D)

\begin{abstract}
Background: The chloroplast of eukaryotic microalgae such as Chlamydomonas reinhardtii is a potential platform for metabolic engineering and the production of recombinant proteins. In industrial biotechnology, inducible expression is often used so that the translation or function of the heterologous protein does not interfere with biomass accumulation during the growth stage. However, the existing systems used in bacterial or fungal platforms do not transfer well to the microalgal chloroplast. We sought to develop a simple inducible expression system for the microalgal chloroplast, exploiting an unused stop codon (TGA) in the plastid genome. We have previously shown that this codon can be translated as tryptophan when we introduce into the chloroplast genome a trnW $W_{\text {UCA }}$ gene encoding a plastidial transfer RNA with a modified anticodon sequence, UCA.

Results: A mutated version of our trnW UCA gene was developed that encodes a temperature-sensitive variant of the tRNA. This allows transgenes that have been modified to contain one or more internal TGA codons to be translated differentially according to the culture temperature, with a gradient of recombinant protein accumulation from $35^{\circ} \mathrm{C}$ (low/off) to $15^{\circ} \mathrm{C}$ (high). We have named this the CITRIC system, an acronym for cold-inducible translational readthrough in chloroplasts. The exact induction behaviour can be tailored by altering the number of TGA codons within the transgene.
\end{abstract}

Conclusions: CITRIC adds to the suite of genetic engineering tools available for the microalgal chloroplast, allowing a greater degree of control over the timing of heterologous protein expression. It could also be used as a heat-repressible system for studying the function of essential native genes in the chloroplast. The genetic components of CITRIC are entirely plastid-based, so no engineering of the nuclear genome is required.

Keywords: Microalgae, Chloroplast, Industrial biotechnology, Inducible expression, tRNA, Premature termination codon, Opal suppression

\footnotetext{
*Correspondence: s.purton@ucl.ac.uk

${ }^{1}$ Algal Research Group, Institute of Structural and Molecular Biology،

University College London, Gower Street, London WC1E 6BT, UK

Full list of author information is available at the end of the article
} 


\section{Background}

The green alga Chlamydomonas reinhardtii is regarded as a safe platform for the expression of recombinant proteins due to its lack of endotoxins and harmful viruses and its excellent performance in recent toxicology tests [1]. Cultivation is easily scaled up and can be carried out in volumes ranging from microplates [2] to photobioreactors and $100 \mathrm{~L}$ bags [3, 4]. Each cell contains a single chloroplast with an average of 83 copies of the circular $205 \mathrm{~kb}$ plastome [5]. In the chloroplast, transgenes can be integrated precisely into the genome by homologous recombination, are present in multiple copies per cell and are not subject to gene silencing effects. A wide range of proteins have been expressed in the $C$. reinhardtii chloroplast, as recently reviewed $[4,6-8]$. These include complex multi-domain immunotoxins for cancer therapy [9], oral vaccine candidates for aquaculture [10], mosquitocidal proteins [11] and a cellulose-hydrolyzing enzyme [12]. This expression platform is capable of producing proteins with disulphide bonds or phosphorylation [13].

Inducible and tunable gene expression systems are frequently used to control the production of recombinant proteins and metabolic products in industrial biotechnology. Such tools are well-developed for both Escherichia coli and yeast (reviewed in $[14,15]$ ) and allow production to be switched on after sufficient biomass has been accumulated, preventing the host transcriptional and translational machinery from being overwhelmed during the growth phase. In the case of metabolic engineering, such control also helps to avoid detrimental effects on the cell such as interference with host metabolic pathways or the accumulation of toxic products or intermediates.

Equivalent inducible and tunable systems are needed for plant and algal chloroplast platforms, and whilst some progress has been made for plants, development is complicated by the cross-talk between the nuclear and plastidial genetic systems.

There are two main mechanisms for the regulation of gene expression in the wild-type $C$. reinhardtii chloroplast, both controlled by nucleus-encoded proteins imported from the cytosol. Firstly, transcriptional regulation is thought to be mediated by a single sigma factor, RPOD, that responds to light levels to establish a circadian rhythm [16]. This contrasts with bacteria and higher plant chloroplasts, which generally use multiple sigma factors to control the transcription of subsets of genes in response to a variety of environmental or endogenous signals; in addition, higher plant chloroplasts have a second RNA polymerase. Secondly, C. reinhardtii regulates the maturation, stabilization (protection from nucleolytic degradation) or translational activation of plastidial transcripts [17]. Many of the nucleus-encoded factors that carry out these processes are highly target-specific; for example, NAC2 binds to the $5^{\prime}$ UTR of $p s b D$ mRNA only. Experimentally decreasing the plastome copy number or the overall transcription rate has little effect on the accumulation of chloroplast-encoded proteins [18, 19].

The development of inducible expression systems for the C. reinhardtii chloroplast has proved more challenging than in bacteria due to this reliance on the nucleus and predominantly post-transcriptional control. In one such system, Nac2 is expressed using the copper-repressible cytochrome $c_{6}$ promoter in the nucleus and the gene of interest is expressed using the $p s b D 5^{\prime}$ UTR in the chloroplast; this enables accumulation of the protein of interest upon removal of copper from the growth medium [20]. Whilst this setup is effective, it requires engineering of both the nuclear and plastidial genomes, and the $5^{\prime}$ UTR of the native $p s b D$ gene must be exchanged in order to maintain constitutive phototrophy. In addition, copper has toxic effects on $C$. reinhardtii $[21,22]$. A variation on this system, using a MetE promoter and Thi4 riboswitch for Nac2, allows control by vitamin $\mathrm{B}_{12}$ or thiamine rather than copper [23, 24]. The only other inducible system published for the $C$. reinhardtii chloroplast is based on the E. coli Lac repressor and requires IPTG [25]. A literature search suggests that this system has not been used in any subsequent projects.

Inducible systems developed for the tobacco chloroplast include a theophylline-dependent translational riboswitch [26, 27] and a nuclear-encoded ethanolinducible T7 polymerase that enables expression of plastid genes under T7 promoters [28]; these tools have yet to be adapted for microalgae.

The use of temperature shifts to induce recombinant protein production is an attractive alternative to these chemical methods, particularly in a commercial context where induction needs to be cheap, simple and achievable at bioreactor scale. Natural isolates of $C$. reinhardtii have been recovered from across eastern North America and more recently from Japan [29, 30]; this species has therefore evolved to tolerate seasonal fluctuations in temperature, making this induction method potentially suitable. Vitova et al. [31] demonstrated that wild-type $C$. reinhardtii can undergo cell division across a temperature range of at least $15-37{ }^{\circ} \mathrm{C}$, with the shortest cell cycles at $20-30{ }^{\circ} \mathrm{C}$. Kremer et al. [32] found the highest growth rate to be at $30^{\circ} \mathrm{C}$, even when cells had been acclimated to 14 or $33^{\circ} \mathrm{C}$. A heat shock response, as measured by the transcript abundance of small heat shock proteins 
(chaperones) after $20 \mathrm{~min}$, is induced at $36{ }^{\circ} \mathrm{C}$ but not $32{ }^{\circ} \mathrm{C}$ [33]. The $H s p 70 A$ heat shock promoter has been used for the inducible expression of nuclear transgenes at $40{ }^{\circ} \mathrm{C}$ [34].

In this paper we demonstrate a new inducible expression system for the C. reinhardtii chloroplast that is based on a method that we developed earlier for preventing transgene expression during cloning and for biocontainment. This method exploits the fact that the UGA stop codon is not used by any of the native genes in the $C$. reinhardtii plastome despite being capable of functioning as a terminator of translation [35], and comprises: (i) a copy of the native trnW (tryptophan tRNA) gene, termed trn $W_{U C A}$ that has an altered anticodon sequence such that the tRNA

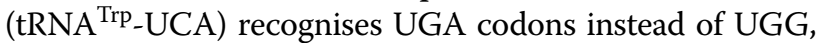
and (ii) a gene of interest with one or more TGG-to-TGA codon alterations. Full-length translation of the gene of interest requires both components to be present, and operates only in $C$. reinhardtii since the chloroplast tRNA is not recognised in E. coli [35].

Here we developed a temperature-sensitive allele of trn$W_{U C A}$ by investigating four different mutations predicted to affect the secondary structure of the tRNA. The first mutation was based on work by Marschalek et al. in 1990 [36], who developed a temperature-sensitive version of a Dictylostelium discoideum glutamic acid tRNA and tested it in Saccharomyces cerevisiae. The authors showed that their C to $\mathrm{U}$ transition in the acceptor stem led to a less stable secondary structure, so the pre-tRNA could be processed to a mature tRNA at $22^{\circ} \mathrm{C}$ but not at $37^{\circ} \mathrm{C}$. We introduced the equivalent mutation into our plastidial $t r n W_{U C A}$ gene. The mutation in our second variant imitates that found in the tryptophan tRNA of a temperature-sensitive $E$. coli isolate [37, 38]. In E. coli, this mutation weakens the acceptor stem and makes it more susceptible to denaturation, especially at elevated temperatures [38]. Our third variant has altered base pairing in the dihydrouracil (D) loop and is based on work on tryptophan variants from E. coli [39]; it should be noted that in E. coli, altering this base (position 24) affected not just the tRNA stability but also the codon-anticodon recognition. Our fourth tRNA design is based purely on the principle that A-U base pairs have weaker hydrogen bonding than G-C pairs, so an alteration in the acceptor stem may destabilize the tRNA secondary structure sufficiently to disable function and/or processing at higher temperatures.

Of these four mutations, we found that the first conferred clear temperature sensitivity upon the synthetic tRNA in the $C$. reinhardtii chloroplast, enabling the development of a cold-inducible and tunable translation system. This eliminates the need for expensive chemical inducers and avoids the risk of contamination during the addition of such reagents.

\section{Results}

\section{Variant 1 of tRNA ${ }^{\text {Trp }}$-UCA $(\mathrm{tCl})$ allows}

temperature-dependent readthrough of internal UGA codons

We designed and tested four variants of the $\operatorname{trn} W_{U C A}$ gene in order to determine whether they would produce a temperature-dependent tRNA in the chloroplast that was still able to recognise internal UGA codons within the transcript of a transgene at a lower temperature, but not at a higher temperature. The variants each had one or two nucleotide changes from our original trn $W_{U C A}$ gene (Fig. 1), with these changes designed to reflect known

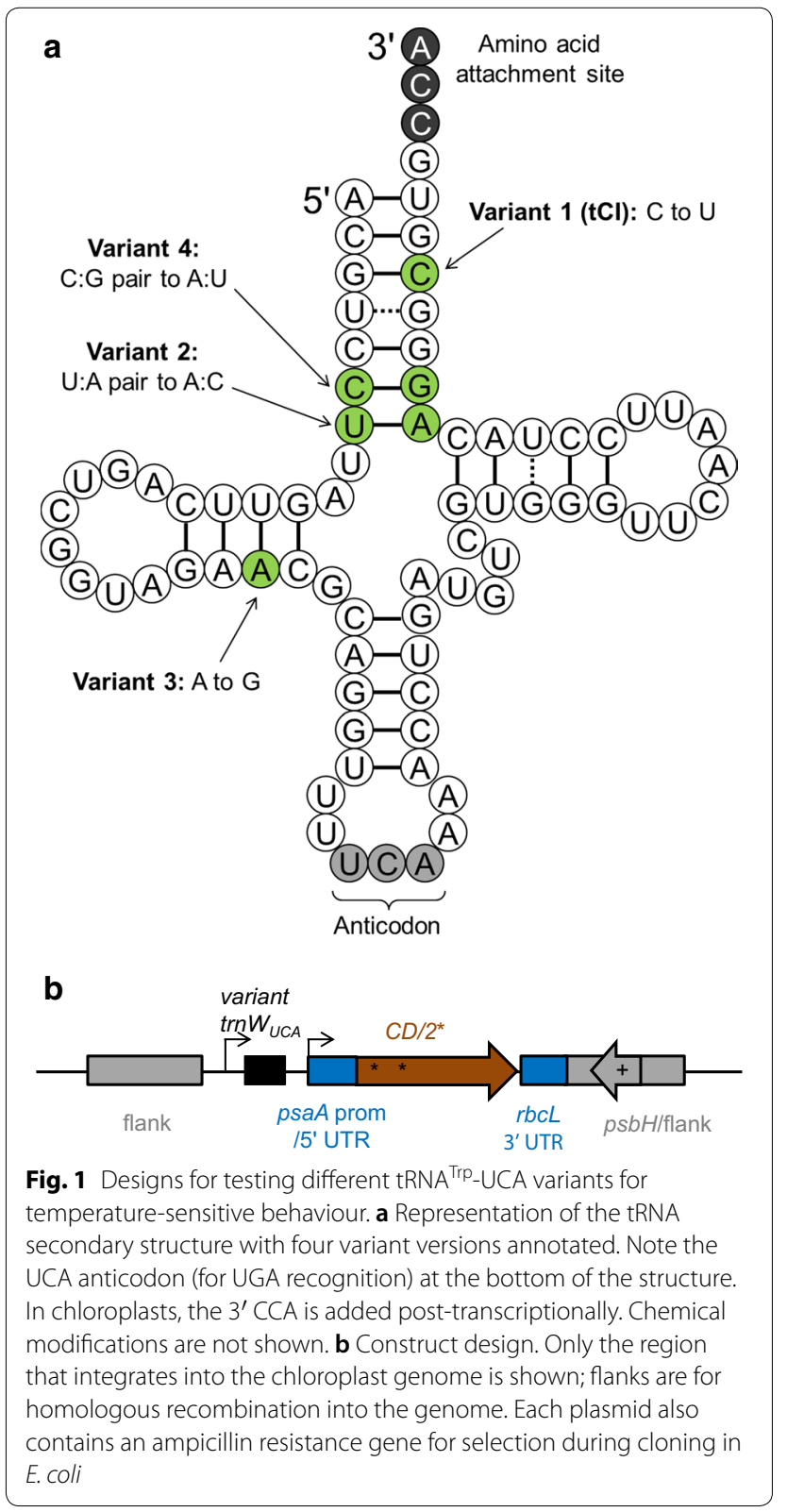


temperature-sensitive mutations in other organisms and/ or a less negative free energy prediction to reduce stability of the RNA secondary structure (see "Background" section). The test transgene was $C D$, a cytosine deaminase gene of bacterial origin but codon-optimised for the $C$. reinhardtii chloroplast. We previously developed $\mathrm{CrCD}$ (encoded by $C D$ ) as a negative selectable marker [40] and chose to use it in the present study for its ease of detection using the C-terminal HA tag, clear phenotype of 5-fluorocytosine sensitivity, and non-toxicity to $E$. coli and C. reinhardtii on normal media.

We generated $C$. reinhardtii cell lines that contained a $C D$ gene with two internal TGG-to-TGA mutations $\left(C D / 2^{*}\right)$ and a $\operatorname{trn} W_{U C A}$ variant gene, both integrated downstream of $p s b H$ in the chloroplast genome (Fig. 1b). Full length $\mathrm{CrCD}$ protein (including the C-terminal HA tag) will only be produced when the tRNA variant allows translational readthrough of the internal UGA codons in the $C D / 2$ * transcript; we tested this by western blot analysis using anti-HA antibodies. The cell lines were grown at $20,25,30$ and $35^{\circ} \mathrm{C}$ for $72 \mathrm{~h}$ and the final level of $\mathrm{CrCD}$ protein was visualised by immunoblotting of whole cell extracts (Fig. 2). Cell lines containing trn $W_{U C A}$ variant genes 2, 3 or 4 did not show marked variation in $\mathrm{CrCD}$ levels across the temperature range, so were not studied further. However, tRNA ${ }^{\operatorname{Tr}}$-UCA variant 1 , which harbours a C-to-U mutation in the acceptor stem, was found to display the desired temperature-dependent behaviour: more $\mathrm{CrCD}$ protein clearly accumulated at the lower temperatures (Fig. 2). Growth tests on media with or without

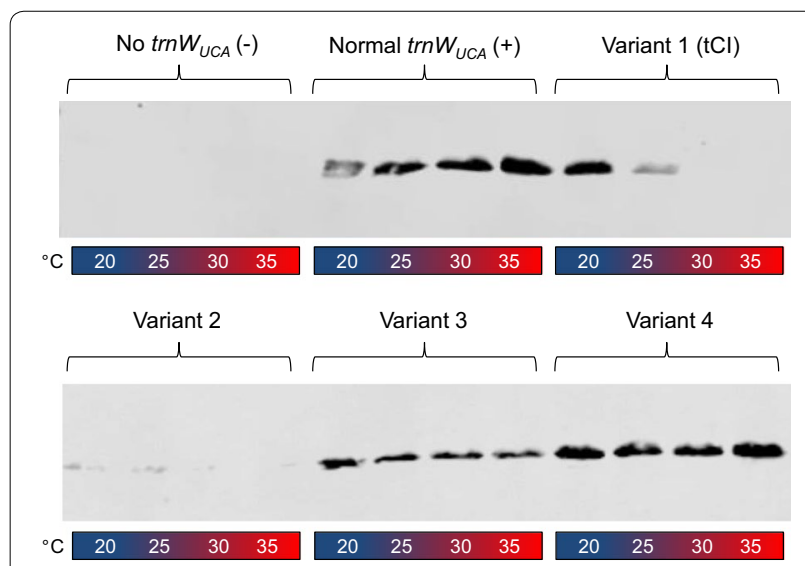

Fig. 2 Analysis of the ability of the four tRNA variants to translate a test protein, $\mathrm{CrCD}$, at different growth temperatures. Six C. reinhardtii cell lines, all containing the $C D / 2^{*}$ gene but with different tRNA variants, were grown for $72 \mathrm{~h}$ at $20,25,30$ or $35^{\circ} \mathrm{C}$. Crude extracts were equalised according to the culture optical density at $750 \mathrm{~nm}$ and subjected to SDS-PAGE and Western blotting using an anti-HA antibody to detect CrCD protein. See Additional file 1:Table S2 for culture conditions 5-fluorocytosine, which CrCD converts to toxic 5-fluorouracil, confirmed this behaviour and demonstrated that the CrCD enzyme produced using tRNA ${ }^{\text {Trp }}$-UCA variant 1 was active (Fig. 3 and Additional file 1: Figure S1). The variant 1 gene was renamed ' $t C l$ ' and its corresponding tRNA ' $t C l$ ' to reflect its cold-inducible behaviour. Growth experiments show that there is no apparent deleterious effect from expressing $t C I$ in $C$. reinhardtii (Additional file 1: Figure S2).

\section{Expression levels can be tailored by varying both induction temperature and the number of internal UGA codons}

To investigate whether the number of UGA codons within an mRNA affected its translational efficiency using tCI, we mutated the $C D / 2 *$ plasmid that contained $t C I$ to create versions with four or six TGG-to-TGA codon changes within the $C D$ gene rather than two (see Sequences 3 and 4 in Additional File 1). These were used to generate $C$. reinhardtii transformants for immunoblotting experiments (Fig. 4a). Increasing the number of internal UGA codons led to a tighter OFF state at $30{ }^{\circ} \mathrm{C}$, with good repression of test protein accumulation at high growth temperatures. This would be suitable for recombinant proteins that are highly toxic to the host cell even at low concentrations. However, the maximum $\mathrm{CrCD}$ protein yield upon induction was lower, suggesting that the amount of active $\mathrm{tCI}$ is rate-limiting when four

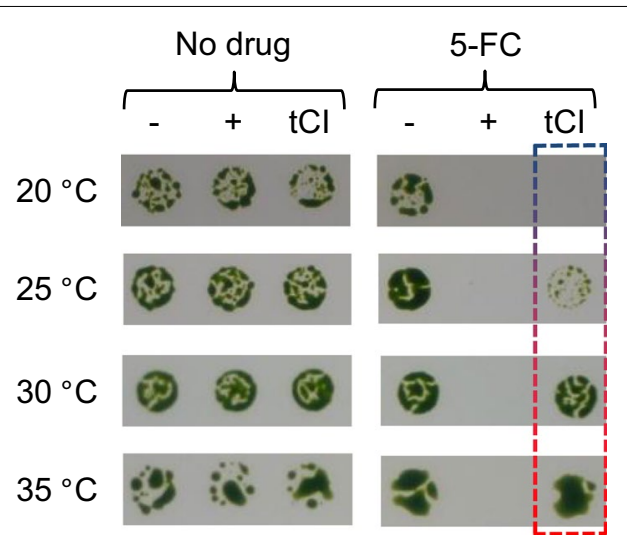

Fig. 3 Growth test demonstrating that the introduction of $t C l$ into the chloroplast genome allows the temperature-dependent translation of functional $\mathrm{CrCD}$ protein from the $\mathrm{CD} / 2^{*}$ gene. Liquid cultures grown at $35^{\circ} \mathrm{C}$ were spotted onto TAP agar containing no drug (left panel) or $2 \mathrm{mg} / \mathrm{ml} 5$-fluorocytosine (5-FC; right panel) then incubated at different temperatures for 10 days. Three $C$. reinhardtii cell lines were used, all containing the $C D / 2^{*}$ gene. In the cell line with no additional tRNA gene $(-), C D / 2^{*}$ is not translated so the cells grow on $5-F C$. In the cell line with $t_{r n} W_{U C A}$ encoding the constitutive version of the tRNA $(+), C D / 2^{*}$ is translated at all growth temperatures and prevents growth on 5-FC. In the cell line with $t C l$, CrCD accumulation and subsequent growth inhibition depend on the temperature 

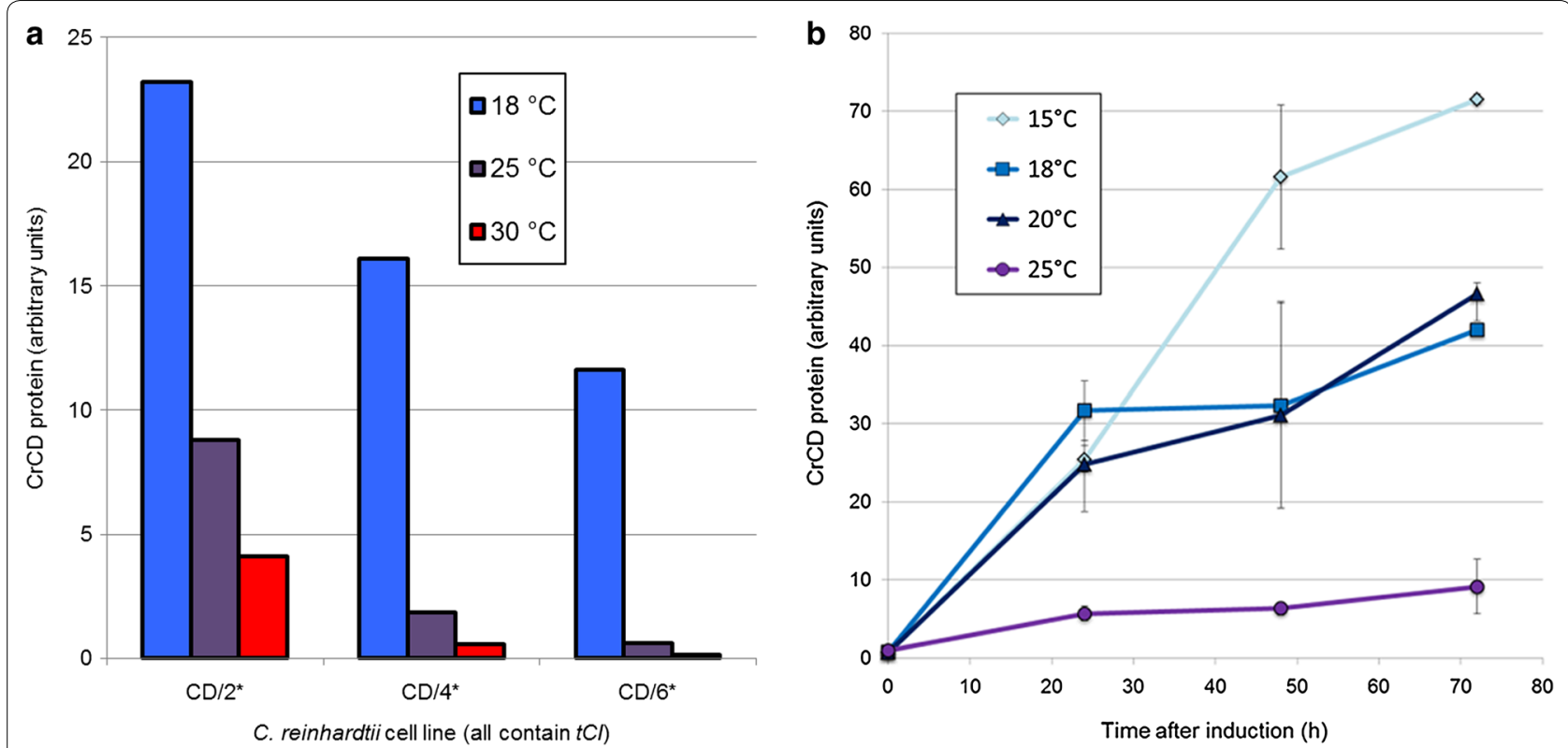

Fig. 4 Investigation of $C r C D$ protein accumulation in C. reinhardtii $t C l$ cell lines upon varying the number of internal TGA codons and/or induction temperature. a Cell lines with 2, 4 or 6 internal TGA codons in the CD gene were pre-cultured at $30^{\circ} \mathrm{C}$ then shifted to 18,25 or $30^{\circ} \mathrm{C}$ for induction. $\mathrm{CrCD}$ protein accumulation was measured by anti-HA immunoblot after $72 \mathrm{~h}$. Equal culture volumes were used for each immunoblot sample, then anti-HA band intensity values were divided by the optical density $(750 \mathrm{~nm}$ ) of that culture to plot relative 'per cell' $\mathrm{CrCD}$. Each bar shows the mean value for two cultures. $\mathbf{b}$ The $C D / 4^{*}$ cell line was grown in four identical flask cultures at $35^{\circ} \mathrm{C}$ to a high cell density $\left(\mathrm{OD}_{750}=2.1\right)$ then induced at $15,18,20$ or $25^{\circ} \mathrm{C}$ using Algem photobioreactors. CrCD accumulation was followed for $72 \mathrm{~h}$ after induction. Samples were equalised according to optical density at $750 \mathrm{~nm}$ and analysed by SDS-PAGE and anti-HA immunoblotting. Error bars (on all data points) show \pm SD for two samples taken from the same flask at each timepoint

or six UGA codons are present. The yield for a particular recombinant protein could therefore be tailored by a combination of genetic element choice (promoter and UTRs), UGA number and induction temperature.

\section{Maximum protein accumulation is achieved at $15^{\circ} \mathrm{C}$}

To determine the best temperature for induction and protein accumulation, the $C D / 4^{*}+t C I$ cell line of $C$. reinhardtii was grown to late exponential phase at $35^{\circ} \mathrm{C}$ ('growth phase') then cooled to a range of temperatures ('induction phase'). The level of CrCD protein accumulation over the next $72 \mathrm{~h}$ was tracked by immunoblotting. As shown in Fig. $4 \mathrm{~b}, \mathrm{CrCD}$ accumulated over the $72 \mathrm{~h}$ under all induction temperatures tested $\left(15-25^{\circ} \mathrm{C}\right)$, but reached the highest levels at $15^{\circ} \mathrm{C}$. A second experiment demonstrated that induction at an even lower temperature of $12{ }^{\circ} \mathrm{C}$ is still efficient but did not improve the $\mathrm{CrCD}$ yield further (Additional file 1: Figure S3; the $C D / 2^{*}+t C I$ cell line was used in this case).

Growth curves following induction are shown in Additional file 1: Figure S4 for the first experiment, and show similar biomass yields reached after approximately $48 \mathrm{~h}$ as the cultures enter stationary phase. The maximum production of the $\mathrm{CrCD}$ protein per unit of biomass is therefore seen following incubation of the culture at $15{ }^{\circ} \mathrm{C}$ for at least $72 \mathrm{~h}$, although the optimum timepoint for harvesting a protein of interest would depend on its stability at the induction temperature, as the protein level depends upon the rate of both translation and degradation.

\section{Inducible production of a cis-abienol synthase enzyme, TPS4}

Inducible systems can be useful in the field of metabolic engineering as they allow the accumulation of cell mass prior to switching on a potentially detrimental enzymatic pathway. For example, some terpenes have cytotoxic effects on the production host (reviewed in [41]). Constitutive expression of the TPS4 gene, encoding a bifunctional cis-abienol synthase from Abies balsamea (Balsam Fir), in the C. reinhardtii chloroplast produces a slight but reproducible growth defect $[42,43]$. This enzyme converts geranylgeranyl diphosphate to cis-abienol, which is a diterpene that can be used as a precursor for Ambrox fragrances [44]. By creating two or four TGG-to-TGA mutations in the codon-optimised TPS4 open reading frame and inserting it alongside $t C I$ into the $C$. reinhardtii chloroplast 
a

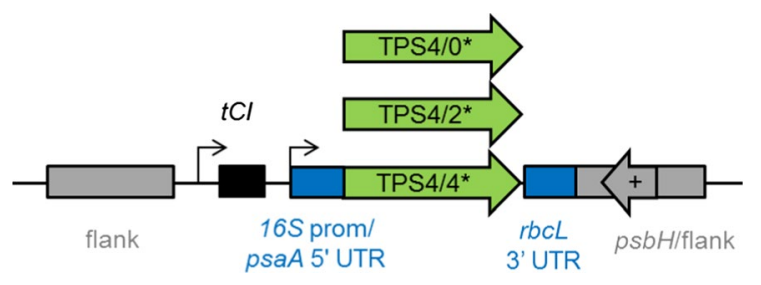

C

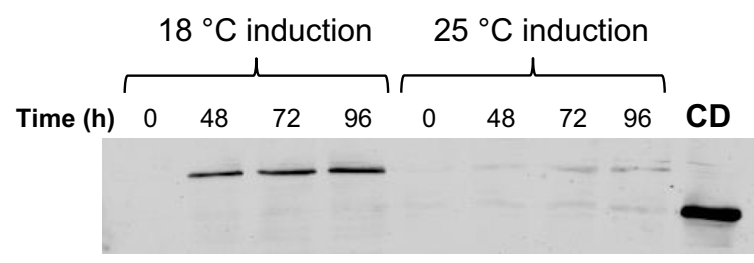

e

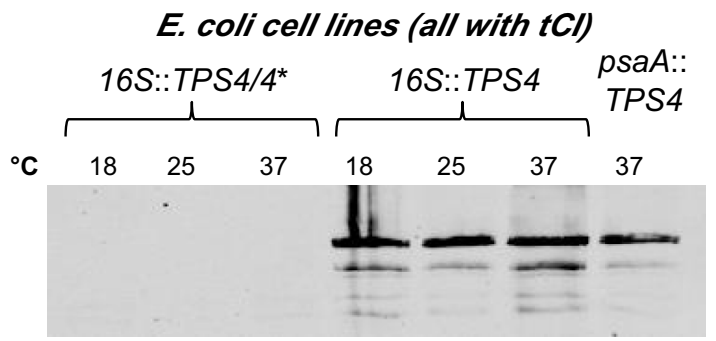

b

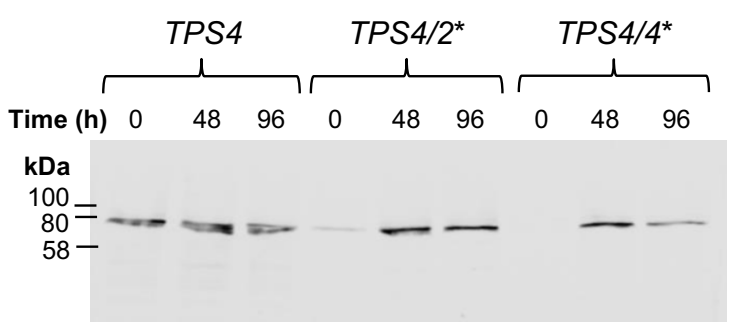

d

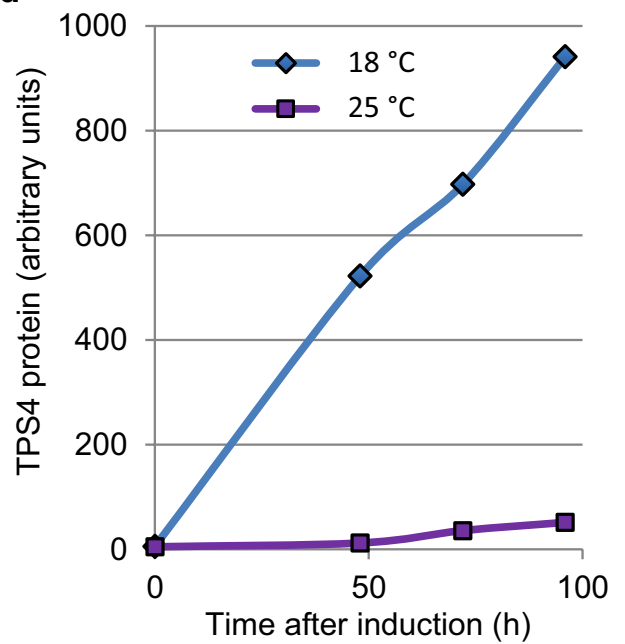

Fig. 5 Cold-inducible synthesis of cis-abienol synthase, TPS4. a Design of chloroplast expression constructs. The TPS4 genes include a C-terminal HA tag for detection. $\mathbf{b}$ Immunoblot using anti-HA antibodies to detect TPS4 protein in C. reinhardtii $\mathrm{tCl}$ cell lines induced at $18^{\circ} \mathrm{C}$. Samples were equalised according to the culture optical density at $750 \mathrm{~nm}$ before loading. The first three lanes show a constitutive TPS4 control cell line with no internal TGA codons. c Anti-HA immunoblot to compare induction temperatures of 18 and $25^{\circ} \mathrm{C}$ in C. reinhardtii. Samples were equalised according to the culture optical density at $750 \mathrm{~nm}$ before loading. A strain synthesising CrCD (right lane) is included as a negative control for the TPS4 band. $\mathbf{d}$ Quantification of the band intensities in part c, showing the TPS4 induction time-course at two temperatures. e Anti-HA immunoblot to detect TPS4 protein in E. coli DH5a containing various TPS4 chloroplast expression plasmids. Total protein stain for this blot is shown in Additional file 1: Figure S6. The incubation temperature is given above each lane

genome (Fig. 5a), we were able to induce TPS4 protein accumulation upon moving the cultures from $30^{\circ} \mathrm{C}$ to $18{ }^{\circ} \mathrm{C}$ (Fig. 5b). Figure 5c, d show the effect of inducing TPS4 at $18{ }^{\circ} \mathrm{C}$ (high expression) versus $25^{\circ} \mathrm{C}$ (low expression) for the $C$. reinhardtii cell line with four internal TGA codons in TPS4.

The C. reinhardtii TPS4 cell lines used for Fig. 5 were developed using our standard glass bead transformation procedure, which involves incubating the minimal media transformation plates at $25{ }^{\circ} \mathrm{C}$ for $2-4$ weeks to allow the selection of phototrophic transformant colonies through restoration of $p s b H$ [45]. However, we found that homoplasmic transformants could also be obtained from plates incubated at $30{ }^{\circ} \mathrm{C}$ (Additional file 1: Figure S5). This could be useful for genes of interest where tighter repression is required during cell line production due to high levels of toxicity.
Informational biocontainment: chloroplast promoters are functional in $\mathrm{E}$. coli, but $\mathrm{tCl}$ is not

We previously demonstrated that when using E. coli as a cloning host, the $C$. reinhardtii psaA promoter and $5^{\prime}$ UTR element allow expression of transgenes in this bacterium $[35,45]$. This is also true for the $C$. reinhardtii $16 \mathrm{~S}$ rRNA promoter plus psaA $5^{\prime}$ UTR combination (Fig. 5e) and is a potential problem when cloning antibacterial or metabolic enzymes that might prove toxic to E. coli. However, the constitutive tRNA ${ }^{\text {Trp }}$-UCA does not function in E. coli, so a construct containing $\operatorname{trn} W_{U C A}$ plus a gene of interest with internal TGA codons will provide protection against transgene expression during cloning in E. coli [35]. Figure $5 \mathrm{e}$ shows that the same holds true for the inducible tRNA, tCI. As this tRNA only functions at low temperatures in $C$. reinhardtii, we allowed $E$. coli to grow 
to mid-log phase $\left(3 \mathrm{~h}\right.$ at $\left.37{ }^{\circ} \mathrm{C}\right)$ then incubated the cultures at a range of temperatures for $18 \mathrm{~h}$; no accumulation of the test protein (TPS4/4*) was observed using anti-HA immunoblotting (Fig. 5e and Additional file 1: Figure S6).

\section{Translational readthrough in the $C$. reinhardtii chloroplast is not inducible by drugs}

Readthrough of premature termination codons (PTCs) is known to be triggered by certain chemicals, including some aminoglycosides, a phenomenon exploited in the search for treatments for PTC-associated genetic diseases such as cystic fibrosis $[46,47]$. Low concentrations of erythromycin and chloramphenicol enhance promiscuous UGA readthrough in E. coli [48]. To investigate whether UGA readthrough could be druginduced in the $C$. reinhardtii chloroplast, we incubated a cell line that contains $C D / 2 *$ but no added tRNA genes in media containing various low concentrations of kanamycin, chloramphenicol, spectinomycin or erythromycin for $48 \mathrm{~h}$. However, we did not observe a significant increase in the level of $\mathrm{CrCD}$ protein above the trace amount seen in the control in any of the conditions tested (Additional file 1: Figure S7). Using the $t C I$ tRNA gene therefore remains the best way to allow inducible readthrough of transgenes containing TGA codons in the C. reinhardtii chloroplast.

\section{Plasmids available for the CITRIC system}

Two plasmids are available for researchers wishing to use the CITRIC system to induce the accumulation of proteins of interest in the C. reinhardtii chloroplast (Fig. 6). Plasmid pWUCA3 carries the cold-inducible trn $W_{U C A}$ gene $(t C I)$ plus an aadA cassette for selection using spectinomycin and streptomycin resistance. This integrates into the chloroplast genome within an intergenic region downstream of $p s a A$ exon 3 . This plasmid can be used in any $C$. reinhardtii cell line; the gene of interest containing internal TGA codons would be introduced separately. Plasmid pWUCA4 contains $t C I$, an intact copy of $p s b H$ for phototrophic selection, and an empty expression cassette into which the gene of interest can be introduced using restriction enzymes SapI and SphI. This plasmid is designed for the transformation of a $p s b H$ deletion mutant such as $C$. reinhardtii TN72 and uses the intergenic region downstream of $p s b H$ as the integration site [45]. DNA sequences of both plasmids are given in Additional file 1 , and the workflow for pWUCA4 is shown in Box 1.

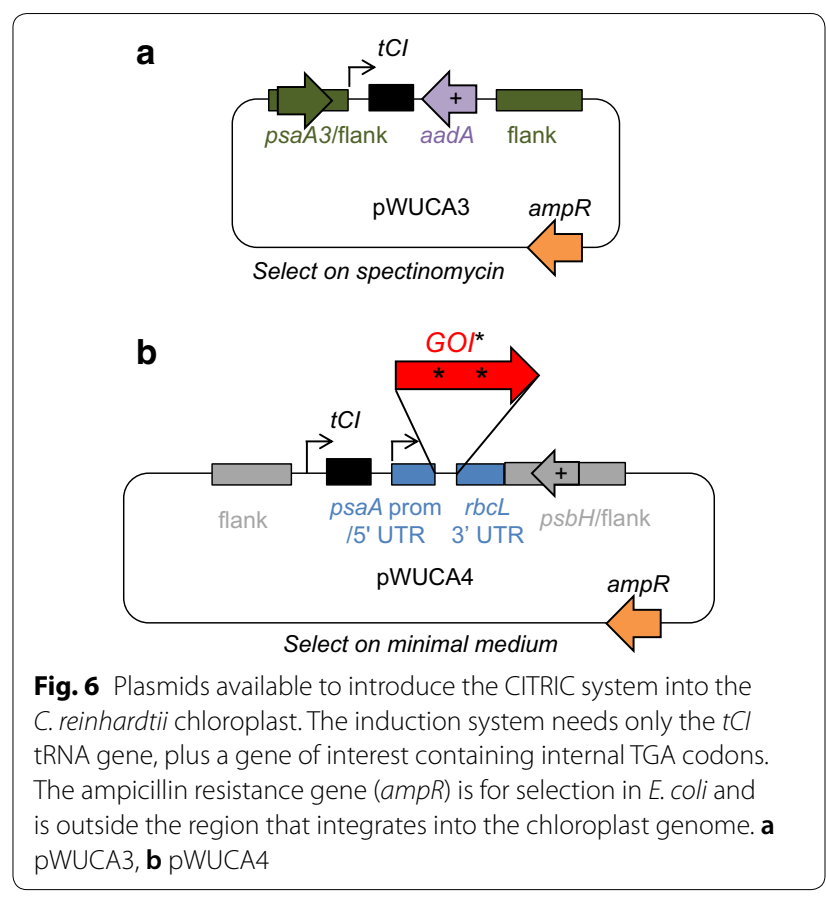

\section{Box 1. Workflow for the CITRIC system: inducible transgene expression}

1. Order the gene of interest, codon-optimised for the C. reinhardtii chloroplast and with 2-6 mutated Trp codons (TGG to TGA), preferably near the N-terminus to reduce translational burden. Include a $\mathrm{C}$-terminal epitope tag if required for protein detection or purification. Include a SapI site at $5^{\prime}$ end for precise fusion to $5^{\prime}$ UTR, and an SphI site at $3^{\prime}$ end.

2. Ligate into empty expression cassette in pWUCA4 using SapI and SphI. Clone in E. coli.

3. Use the resulting plasmid to transform $C$. reinhardtii TN72. Select on minimal media at $25^{\circ} \mathrm{C}$ (or $30{ }^{\circ} \mathrm{C}$ if protein considered highly toxic) for restoration of phototrophy due to $p s b H$ gene in pWUCA4. Colonies appear in 2-4 weeks.

4. Confirm transgene integration and homoplasmy by PCR.

5. Identify optimal temperatures for the growth and induction phases. This may depend on the transgene and number of TGA codons used, but is likely to be $30-35{ }^{\circ} \mathrm{C}$ for the growth (repression) phase and $15-20^{\circ} \mathrm{C}$ for full induction.

N.B. The system can be adapted for other plasmids and $C$. reinhardtii cell lines. Only the modified gene of interest, the $t C I$ gene and a selectable marker are required. 


\section{Discussion}

Four variants of $\mathrm{tRNA}^{\mathrm{Trp}}$-UCA were tested for their function at different temperatures (Figs. 1 and 2). Structural stability and correct folding of the tRNA are prerequisites for functionality. In silico secondary structure predictions using RNAfold software [49] shed some light on the observed results. First, the free energy prediction of variant 1 (tCI) is the least negative, indicating that this is the least stable of all the variants tested (Additional file 1: Figure S8). This is likely to contribute to its loss of function at elevated temperatures, either directly through unfolding or through an inability to fold correctly for pre-tRNA processing, making it useful for the CITRIC system. Second, whilst the structure predicted to have the minimum free energy was a classic tRNA cloverleaf shape for all combinations of tRNA and temperature shown in Additional file 1: Figure S8, the acceptor stem in variant 2 consisted of only six base pairings instead of seven. This is due to the mutation creating a non WatsonCrick match (Fig. 1a); an equivalent mutation creates a thermolabile tryptophan tRNA in E. coli [37], but appears to almost ablate function in $C$. reinhardtii (Fig. 2).

CITRIC could be used for the production of proteins (vaccines, antibodies, hormones, etc.) or for metabolic engineering. If the product of interest is the induced protein itself, this can be harvested after the cold induction phase. Indeed, temperatures of $6-25^{\circ} \mathrm{C}$ are used in chemically-induced $E$. coli protein expression systems to aid protein stability and/or solubility [15]. However, if the induced protein is an enzyme for metabolic engineering within the chloroplast, the optimum temperatures for substrate availability, enzyme activity and product stability must be taken into account. For some reactions, sufficient product may be obtained during the cold induction phase, whereas for others a warmer incubation phase may be required following induction. Enzymes originally found in psychrophiles (cold-adapted organisms) are more likely to have efficient low-temperature activity and could be useful in this context $[50,51]$.

The generation of cell lines in which a certain essential endogenous gene is under temperature regulation can be valuable for assigning function [52], as constitutive knockout of such genes is not possible. The CITRIC system could be used to study chloroplast genes of unknown function such as orf1995, a large open reading frame for which there is expression evidence at both the transcript and protein level [5]. Such experiments will be easier now that a more accurate $C$. reinhardtii chloroplast genome sequence is available [5].

Another potential use for this system is the generation of markerless chloroplast transformants in any cell line. A constitutive selectable marker together with an inducible counter-selectable marker would be flanked by direct repeats, and selection carried out at the uninduced temperature. Once homoplasmy is established, translation of the counter-selectable marker would be cold-induced to select for homologous recombination between the repeats and loss of both marker genes. A similar strategy has been employed in E. coli using an IPTG-inducible $c c d B$ toxin gene [53]. Possible alternatives to $c c d B$ include the MazF endoribonuclease family [54-56] or toxin genes from toxin-antitoxin systems [57].

The native $C$. reinhardtii chloroplast tRNA ${ }^{\text {Trp }}$ encoded by $\operatorname{trn} W$ translates UGG codons as tryptophan. The tRNA ${ }^{\text {Trp }}$-UCA version of it that we developed previously has a mutated anticodon so that it recognises UGA codons (Fig. 1a); we inferred that this tRNA still adds tryptophan, as it was able to rescue a TGG-to-TGA mutation that affected an essential tryptophan residue in PsaA [35]. The tCI tRNA used in the CITRIC system has one further mutation, namely a C-to-U transition in the acceptor stem at position 70 (Fig. 1a). We are confident that the majority of residues incorporated at CITRIC TGA sites are tryptophan, as purified $\mathrm{CrCD}$ protein from a $C$. reinhardtii $C D / 6$ * tCI strain (where 6 of the 7 TGG codons in $C D$ are replaced with TGA) gives an equivalent strength signal with a tryptophan-specific binding compound when compared to control CrCD protein (Additional file 1: Figure S9). Nevertheless, the recognition of tRNAs by their cognate aminoacyl tRNA synthetases (i.e. amino acid specificity) can be complex [58], so it is recommended that the amino acid sequences of proteins expressed using the CITRIC system are verified if incorporation of $100 \%$ tryptophan at UGA codon positions is required.

It should be noted that internal UGA readthrough has been observed by others in E. coli when a strong inducible bacterial promoter, single UGA codon and deliberately readthrough-enhancing RNA context is used (e.g. placing a GAC codon two positions upstream) [59]. The use of chloroplast promoters and inclusion of more than one internal UGA codon in the gene of interest will help to prevent readthrough in $E$. coli when using CITRIC, but this should be confirmed experimentally for each gene of interest if a lack of readthrough is being relied upon for biocontainment.

The possibilities and limitations of adapting the artificial tRNA system for other microalgal or higher plant chloroplasts was discussed previously [35]. In brief, this depends on the availability of free or rarely-used codons in the chloroplast genome, in addition to the existence of plastid transformation technology for that species. The same criteria apply for the CITRIC system. 


\section{Conclusions}

We have developed a cold-inducible translation system for the $C$. reinhardtii chloroplast using a tRNA that can read through internal UGA codons at low temperatures only. This increases the scope of the organism as a platform for heterologous protein production, metabolic engineering and the study of essential genes. We hope that it will prove a useful addition to the genetic engineering toolbox for microalgae.

\section{Methods}

Algal strains, culture conditions and transformation

All $C$. reinhardtii cell lines used in this research were made from the parental line TN72 [40], which is a $c w 15$ (i.e. cell wall deficient) mutant with an $\operatorname{aadA}$ spectinomycin resistance cassette replacing part of $p s b H$ in the chloroplast genome. TN72 is available from the Chlamydomonas Resource Center (http://www.chlamycoll ection.org) as strain CC-5168. Transformation of TN72 was performed using the glass bead vortexing method [40] and a plasmid containing an intact copy of $p s b H$ in addition to the genes of interest, resulting in markerless phototrophic transformants whose homoplasmicity was confirmed by PCR as described previously [35]. DNA sequencing (Source BioScience, Nottingham, UK) was used to confirm that the introduced genetic elements were intact. C. reinhardtii was grown in Trisacetate phosphate (TAP) medium [60], with 2\% agar (Fisher Bioreagents, New Hampshire, USA) added for solid plates. For selection of phototrophic transformants, high-salt minimal (HSM) medium with $2 \%$ agar was used [60]. Liquid cultures were grown in glass flasks or in clear plastic 12 well tissue culture plates (VWR, Pennsylvania, USA). For some experiments (see Additional file 1: Table S2), two parallel Algem photobioreactors were used (Algenuity, Stewartby, UK).

\section{Plasmid construction}

To test trn $W_{U C A}$ variant genes 1 to 4 (see Sequence 2 in Additional file 1), certain single or double point mutations were introduced into the trn $W_{U C A}$ region of plasmid pWUCA2-CD* [35] by PCR amplification using the primers in Additional file 1: Table S1 followed by Gibson assembly [61] and cloning using chemically competent $E$. coli DH5 $\alpha$ [62]. Plasmid pWUCA4 was prepared similarly from pWUCA2 [35], introducing a single point mutation to change $t r n W_{U C A}$ to $t C I$. The same applies to pWUCA3, created from pWUCA1 [35]. To create the TPS4 plasmids in Fig. 5a, a TPS4 cis-abienol synthase gene with a C-terminal HA tag and codon optimisation for the $C$. reinhardtii chloroplast was obtained from Julie Zedler (University of Kent, UK) and cloned between the SapI and SphI sites in pWUCA4. The $p s a A$ exon 1 promoter was replaced with a $16 \mathrm{~S}$ rRNA promoter amplified from C. reinhardtii using Gibson assembly; see Sequence 7 in Additional file 1 . The psaA 5' UTR was retained. The $C$. reinhardtii $16 \mathrm{~S}$ rRNA promoter has been shown by others to give high expression levels for other transgenes [12, 63].

Internal stop codons (TGG-to-TGA mutations) were introduced into $C D$ and TPS4 at the positions indicated in the supplementary sequence data (Additional file 1): the relevant plasmid was amplified using primers spanning each mutation site in the format $\mathrm{N}_{10}(\mathrm{G}$ to A) $\mathrm{N}_{13-20}$ for the forward primer and $\mathrm{N}_{10}(\mathrm{C}$ to $\mathrm{T}) \mathrm{N}_{13-20}$ for the reverse primer, and the PCR products were reassembled using Gibson assembly. All PCR amplification was carried out using Phusion High-Fidelity DNA Polymerase, and plasmids were prepared for algal transformation using a GeneJET Plasmid Midiprep Kit (both ThermoFisher, Waltham, USA).

\section{5-fluorocytosine sensitivity test}

For the growth test in Fig. 3, $20 \mathrm{ml}$ TAP cultures of the three $C$. reinhardtii cell lines were grown at $35{ }^{\circ} \mathrm{C}$ for $48 \mathrm{~h}$. Cultures were then diluted to $\mathrm{OD}_{750}=0.2$ and $5 \mu \mathrm{l}$ was spotted onto TAP $+2 \%$ agar plates that contained 0 or $2 \mathrm{mg} / \mathrm{ml}$ 5-fluorocytosine, which was prepared as a $5 \mathrm{X}$ stock in TAP medium. Plates were incubated under $25 \mu \mathrm{E} / \mathrm{m}^{2} / \mathrm{s}$ light at $20,25,30$ or $35^{\circ} \mathrm{C}$ and photographed after 10 days.

\section{C. reinhardtii immunoblots}

Growth conditions for each induction experiment are given in Additional file 1: Table S2. Cells were harvested by centrifugation $(4000 \mathrm{~g}, 5 \mathrm{~min}$ ) and resuspended in $0.8 \mathrm{M}$ Tris/ $\mathrm{HCl} \mathrm{pH} \mathrm{8.3,0.2} \mathrm{M} \mathrm{sorbitol} \mathrm{and}$ $1 \% \beta$-mercaptoethanol to equal cell densities, as measured by light scattering of the cell culture at $750 \mathrm{~nm}$. The exceptions to this were Fig. $4 \mathrm{a}$ and Additional file 1: Figure S7, where equal culture volumes were used instead (see figure legends). SDS-PAGE, blotting onto nitrocellulose membranes and antibody probing were performed as described previously [35], i.e. all membranes were probed with 1:5000 rabbit $\alpha \mathrm{HA}$ primary antibody (Sigma-Aldrich, Missouri, USA) then 1:25,000 goat $\alpha$-rabbit Dylight 800 secondary antibody (ThermoFisher). HA-tagged CrCD and TPS4 proteins were then detected using an Odyssey CLx imaging system at $800 \mathrm{~nm}$ and analysed in Image Studio (both LI-COR, Nebraska, USA).

\section{E. coli immunoblot}

Escherichia coli DH5 $\alpha$ cultures containing each of the three TPS4 plasmids were grown in $5 \mathrm{ml} \mathrm{LB}$ with $100 \mu \mathrm{g} / \mathrm{ml}$ ampicillin for $3 \mathrm{~h}$ at $37^{\circ} \mathrm{C}$. Optical densities 
were then adjusted to 0.75 at $600 \mathrm{~nm}$, and $1 \mathrm{ml}$ cultures were transferred to 18,25 or $37{ }^{\circ} \mathrm{C}$ incubators, shaking at $200 \mathrm{rpm}$ for $18 \mathrm{~h}$. The final optical densities at $600 \mathrm{~nm}$ were in the range 2.5-6.0 depending on temperature. Cultures were pelleted and resuspended in sample buffer to equal optical densities following the Mini-PROTEAN Tetra Cell manual (Bio-Rad, California, USA). $200 \mu \mathrm{l}$ samples were boiled for $2 \mathrm{~min}$ and centrifuged for $2 \mathrm{~min}$ at $13,000 \mathrm{~g}$, then $10 \mu \mathrm{l}$ of the supernatant was separated on a $15 \%$ acrylamide SDSPAGE gel (100 V, $150 \mathrm{~min})$. The gel was blotted onto a Hybond ECL nitrocellulose membrane (GE Healthcare, Illinois, USA) using a Trans-blot semi-dry transfer cell (Bio-Rad) run at $20 \mathrm{~V}$ for $1 \mathrm{~h}$. The membrane was stained using REVERT Total Protein Stain (LICOR) and imaged at $700 \mathrm{~nm}$ to confirm equal loading and transfer. After blocking overnight at $4{ }^{\circ} \mathrm{C}$ in TBS-T $+5 \%$ milk, the membrane was probed and imaged as above except that the primary antibody concentration was $1: 25,000$.

\section{Additional file}

Additional file 1: Figure S1. Testing tRNA $A^{\text {Trp }}$-UCA variants for temperature-dependent behaviour. Figure S2. Growth curve of $C$. reinhardtii cell lines, showing that the temperature-sensitive tRNA (tCl) does not cause a growth defect. Figure S3. Induction of CrCD protein in the C. reinhardtii $\mathrm{CD} / 2^{*}+t \mathrm{Cl}$ cell line at low temperatures. Figure $\mathbf{S} 4$. Growth curves for C. reinhardtii $C D / 4^{*}+t C l$ following induction at $15-25^{\circ} \mathrm{C}$. Figure $\mathrm{S} 5$. $\mathrm{PCR}$ confirming homoplasmic transgene integration into C. reinhardtii TN72 using transformation plates incubated at $30^{\circ} \mathrm{C}$. Figure S6. REVERT total protein staining of E. coli western blot membrane in Fig. 5e, confirming equal loading and blotting across lanes. Figure S7. Investigation of readthrough effect of four drugs as an alternative method of induction. Figure S8. Minimum free energy calculations for each tRNA variant at $15-35^{\circ} \mathrm{C}$. Figure S9. Detection of tryptophan in purified $\mathrm{CrCD}$ protein from C. reinhardtii CD (positive control) and $C D / 6^{*}+t C l$ cell lines. Table $\mathbf{S 1}$. Primers used to alter trnW UCA to make variants 1 to 4 . Table S2. Conditions for $C$. reinhardtii induction experiments. Sequences 1 to 9 . Includes DNA sequences for tRNAs, genes CD and TPS4, 16S promoter and plasmids PWUCA3 and PWUCA4.

\section{Abbreviations}

5-FC: 5-fluorocytosine; CITRIC: cold-inducible translational readthrough in chloroplasts; CrCD: cytosine deaminase protein; PTC: premature termination codon; TAP: tris-acetate-phosphate medium; tCl: tRNA ${ }^{\text {TrP }}$-UCA variant 1 (coldinducible); $t C l$ : $\operatorname{trn} W_{U C A}$ variant 1 gene.

\section{Authors' contributions}

SP and RY conceived the ideas for this study. RY performed the experiments and wrote the manuscript. Both authors read and approved the final manuscript.

\section{Author details}

${ }^{1}$ Algal Research Group, Institute of Structural and Molecular Biology, University College London, Gower Street, London WC1E 6BT, UK. ${ }^{2}$ Present Address: Department of Medicine, Sir Alexander Fleming Building, Imperial College London, South Kensington Campus, London SW7 2AZ, UK.

\section{Acknowledgements}

We thank Julie Zedler (University of Kent) for the TPS4 plasmid and Vinzenz Bayro Kaiser (Tel Aviv University) for advice on C. reinhardtii cultivation at high temperatures.

\section{Competing interests}

The authors declare that they have no competing interests.

\section{Availability of data and materials}

All data generated or analysed during this study are included in this published article and its additional files. C. reinhardtii TN72 and plasmids pWUCA3 and pWUCA4 are available from the Chlamydomonas Resource Center (http:// www.chlamycollection.org).

\section{Consent for publication}

Not applicable.

\section{Ethics approval and consent to participate}

Not applicable.

\section{Funding}

This study was funded by the UK's Biotechnology and Biological Sciences Research Council; Grant BB/L002957/1.

\section{Publisher's Note}

Springer Nature remains neutral with regard to jurisdictional claims in published maps and institutional affiliations.

Received: 6 September 2018 Accepted: 16 November 2018 Published online: 24 November 2018

\section{References}

1. Murbach TS, Glavits R, Endres JR, Hirka G, Vertesi A, Beres E, Szakonyine IP. A toxicological evaluation of Chlamydomonas reinhardtii, a green algae. Int J Toxicol. 2018;37:53-62.

2. Haire TC, Bell C, Cutshaw K, Swiger B, Winkelmann K, Palmer AG. Robust microplate-based methods for culturing and in vivo phenotypic screening of Chlamydomonas reinhardtii. Front Plant Sci. 2018;9:235.

3. Gimpel JA, Hyun JS, Schoepp NG, Mayfield SP. Production of recombinant proteins in microalgae at pilot greenhouse scale. Biotechnol Bioeng. 2015;112:339-45.

4. Taunt HN, Stoffels L, Purton S. Green biologics: the algal chloroplast as a platform for making biopharmaceuticals. Bioengineered. 2018;9:48-54.

5. Gallaher SD, Fitz-Gibbon ST, Strenkert D, Purvine SO, Pellegrini M, Merchant SS. High-throughput sequencing of the chloroplast and mitochondrion of Chlamydomonas reinhardtii to generate improved de novo assemblies, analyze expression patterns and transcript speciation, and evaluate diversity among laboratory strains and wild isolates. Plant $\mathrm{J}$. 2018;93:545-65.

6. Rasala BA, Mayfield SP. Photosynthetic biomanufacturing in green algae; production of recombinant proteins for industrial, nutritional, and medical uses. Photosynth Res. 2015;123:227-39.

7. Specht EA, Mayfield SP. Algae-based oral recombinant vaccines. Front Microbiol. 2014;5:60.

8. Shamriz S, Ofoghi H. Outlook in the application of Chlamydomonas reinhardtii chloroplast as a platform for recombinant protein production. Biotechnol Genet Eng Rev. 2016;32:92-106.

9. Tran M, Van C, Barrera DJ, Pettersson PL, Peinado CD, Bui J, Mayfield SP. Production of unique immunotoxin cancer therapeutics in algal chloroplasts. Proc Natl Acad Sci U S A. 2013;110:E15-22.

10. Charoonnart P, Purton S, Saksmerprome V. Applications of microalgal biotechnology for disease control in aquaculture. Biology (Basel). 2018;7:24.

11. Kang $\mathrm{S}$, Odom OW, Malone CL, Thangamani S, Herrin DL. Expression of a synthetic gene for the major cytotoxin (Cyt1 Aa) of Bacillus thuringiensis subsp. israelensis in the chloroplast of wild-type Chlamydomonas. Biology (Basel). 2018;7:29. 
12. Richter LV, Yang H, Yazdani M, Hanson MR, Ahner BA. A downstream box fusion allows stable accumulation of a bacterial cellulase in Chla mydomonas reinhardtii chloroplasts. Biotechnol Biofuels. 2018;11:133.

13. Ravi A, Guo S, Rasala B, Tran M, Mayfield S, Nikolov ZL. Separation options for phosphorylated osteopontin from transgenic microalgae Chlamydomonas reinhardtii. Int J Mol Sci. 2018;19:585.

14. Besada-Lombana PB, McTaggart TL, Da Silva NA. Molecular tools for pathway engineering in Saccharomyces cerevisiae. Curr Opin Biotechnol. 2017:53:39-49.

15. Jia B, Jeon CO. High-throughput recombinant protein expression in Escherichia coli: current status and future perspectives. Open Biol. 2016;6:160196

16. Carter ML, Smith AC, Kobayashi H, Purton S, Herrin DL. Structure, circadian regulation and bioinformatic analysis of the unique sigma factor gene in Chlamydomonas reinhardtii. Photosynth Res. 2004;82:339-49.

17. Cavaiuolo M, Kuras R, Wollman FA, Choquet Y, Vallon O. Small RNA profiling in Chlamydomonas: insights into chloroplast RNA metabolism. Nucleic Acids Res. 2017:45:10783-99.

18. Karcher D, Koster D, Schadach A, Klevesath A, Bock R. The Chlamydomonas chloroplast HLP protein is required for nucleoid organization and genome maintenance. Mol Plant. 2009;2:1223-32.

19. Eberhard S, Drapier D, Wollman FA. Searching limiting steps in the expression of chloroplast-encoded proteins: relations between gene copy number, transcription, transcript abundance and translation rate in the chloroplast of Chlamydomonas reinhardtii. Plant J. 2002;31:149-60.

20. Surzycki R, Cournac L, Peltier G, Rochaix JD. Potential for hydrogen production with inducible chloroplast gene expression in Chlamydomonas. Proc Natl Acad Sci U S A. 2007;104:17548-53.

21. Jamers A, Blust R, De Coen W, Griffin JL, Jones OA. Copper toxicity in the microalga Chlamydomonas reinhardtii: an integrated approach. Biometals. 2013:26:731-40.

22. Jiang Y, Zhu Y, Hu Z, Lei A, Wang J. Towards elucidation of the toxic mechanism of copper on the model green alga Chlamydomonas reinhardtii. Ecotoxicology. 2016;25:1417-25.

23. Ramundo S, Rochaix JD. Controlling expression of genes in the unicellular alga Chlamydomonas reinhardtii with a vitamin-repressible riboswitch. Methods Enzymol. 2015;550:267-81.

24. Helliwell KE, Scaife MA, Sasso S, Araujo AP, Purton S, Smith AG. Unraveling vitamin B12-responsive gene regulation in algae. Plant Physiol. 2014;165:388-97.

25. Kato K, Marui T, Kasai S, Shinmyo A. Artificial control of transgene expression in Chlamydomonas reinhardtii chloroplast using the lac regulation system from Escherichia coli. J Biosci Bioeng. 2007;104:207-13.

26. Verhounig A, Karcher D, Bock R. Inducible gene expression from the plastid genome by a synthetic riboswitch. Proc Natl Acad Sci U S A. 2010;107:6204-9.

27. Emadpour M, Karcher D, Bock R. Boosting riboswitch efficiency by RNA amplification. Nucleic Acids Res. 2015;43:e66.

28. Lossl A, Bohmert K, Harloff H, Eibl C, Muhlbauer S, Koop HU. Inducible trans-activation of plastid transgenes: expression of the R. eutropha phb operon in transplastomic tobacco. Plant Cell Physiol. 2005;46:1462-71.

29. Proschold T, Harris EH, Coleman AW. Portrait of a species: Chlamydomonas reinhardtii. Genetics. 2005;170:1601-10.

30. Nakada T, Shinkawa H, Ito T, Tomita M. Recharacterization of Chlamydomonas reinhardtii and its relatives with new isolates from Japan. J Plant Res. 2010;123:67-78.

31. Vitova M, Bisova K, Hlavova M, Kawano S, Zachleder V, Cizkova M. Chlamydomonas reinhardtii: duration of its cell cycle and phases at growth rates affected by temperature. Planta. 2011;234:599-608.

32. Kremer CT, Fey SB, Arellano AA, Vasseur DA. Gradual plasticity alters population dynamics in variable environments: thermal acclimation in the green alga Chlamydomonas reinhardtii. Proc Biol Sci. 2018;285:20171942.

33. Kobayashi Y, Harada N, Nishimura Y, Saito T, Nakamura M, Fujiwara T, Kuroiwa T, Misumi O. Algae sense exact temperatures: small heat shock proteins are expressed at the survival threshold temperature in Cyanidioschyzon merolae and Chlamydomonas reinhardtii. Genome Biol Evol. 2014;6:2731-40

34. Shao N, Bock R. A codon-optimized luciferase from Gaussia princeps facilitates the in vivo monitoring of gene expression in the model alga Chlamydomonas reinhardtii. Curr Genet. 2008;53:381-8.
35. Young RE, Purton S. Codon reassignment to facilitate genetic engineering and biocontainment in the chloroplast of Chlamydomonas reinhardtii. Plant Biotechnol J. 2016:14:1251-60.

36. Marschalek R, Kalpaxis D, Dingermann T. Temperature sensitive synthesis of transfer RNAs in vivo in Saccharomyces cerevisiae. EMBO J. 1990;9:1253-8

37. Eisenberg SP, Soll L, Yarus M. The purification and sequence of a temperature-sensitive tryptophan tRNA. J Biol Chem. 1979;254:5562-6.

38. Eisenberg SP, Yarus M. The structure and aminoacylation of a temperature-sensitive tRNATrp (Escherichia coli). J Biol Chem. 1980;255:1128-37.

39. Hirsh D. Tryptophan transfer RNA as the UGA suppressor. J Mol Biol. 1971;58:439-58.

40. Young RE, Purton S. Cytosine deaminase as a negative selectable marker for the microalgal chloroplast: a strategy for the isolation of nuclear mutations that affect chloroplast gene expression. Plant J. 2014;80:915-25.

41. Kemper K, Hirte M, Reinbold M, Fuchs M, Bruck T. Opportunities and challenges for the sustainable production of structurally complex diterpenoids in recombinant microbial systems. Beilstein J Org Chem. 2017;13:845-54.

42. Zedler JA, Gangl D, Guerra T, Santos E, Verdelho W, Robinson C. Pilot-scale cultivation of wall-deficient transgenic Chlamydomonas reinhardtii strains expressing recombinant proteins in the chloroplast. Appl Microbiol Biotechnol. 2016;100:7061-70.

43. Zedler JA, Gangl D, Hamberger B, Purton S, Robinson C. Stable expression of a bifunctional diterpene synthase in the chloroplast of Chlamydomonas reinhardtii. J Appl Phycol. 2015;27:2271-7.

44. Zerbe $P$, Chiang A, Yuen M, Hamberger B, Hamberger B, Draper JA, Britton $\mathrm{R}$, Bohlmann J. Bifunctional cis-abienol synthase from Abies balsamea discovered by transcriptome sequencing and its implications for diterpenoid fragrance production. J Biol Chem. 2012;287:12121-31.

45. Wannathong T, Waterhouse JC, Young RE, Economou CK, Purton S. New tools for chloroplast genetic engineering allow the synthesis of human growth hormone in the green alga Chlamydomonas reinhardtii. Appl Microbiol Biotechnol. 2016;100:5467-77.

46. Bordeira-Carrico R, Pego AP, Santos M, Oliveira C. Cancer syndromes and therapy by stop-codon readthrough. Trends Mol Med. 2012;18:667-78.

47. Liang F, Shang H, Jordan NJ, Wong E, Mercadante D, Saltz J, Mahiou J, Bihler HJ, Mense M. High-throughput screening for readthrough modulators of CFTR PTC mutations. SLAS Technol. 2017;22:315-24

48. Thompson J, Pratt CA, Dahlberg AE. Effects of a number of classes of $50 \mathrm{~S}$ inhibitors on stop codon readthrough during protein synthesis. Antimicrob Agents Chemother. 2004:48:4889-91.

49. Lorenz R, Bernhart SH, Zu Siederdissen CH, Tafer H, Flamm C, Stadler PF, Hofacker IL. ViennaRNA Package 2.0. Algorithms Mol Biol. 2011;6:26.

50. Cavicchioli R, Charlton T, Ertan H, Mohd Omar S, Siddiqui KS, Williams TJ. Biotechnological uses of enzymes from psychrophiles. Microb Biotechnol. 2011:4:449-60.

51. Siddiqui KS, Williams TJ, Wilkins D, Yau S, Allen MA, Brown MV, Lauro FM, Cavicchioli R. Psychrophiles. Annu Rev Earth Planet Sci. 2013:41:87-115.

52. Breker M, Lieberman K, Tulin F, Cross FR. High-throughput robotically assisted isolation of temperature-sensitive lethal mutants in Chlamydomonas reinhardtii. J Vis Exp. 2016. https://doi.org/10.3791/54831.

53. Zhang Q, Yan Z, Xu Y, Sun J, Shang G. Characterization of inducible ccdB gene as a counterselectable marker in Escherichia coli recombineering. Curr Microbiol. 2017:74:961-4.

54. Shimazu T, Degenhardt K, Nur EKA, Zhang J, Yoshida T, Zhang Y, Mathew R, White E, Inouye M. NBK/BIK antagonizes MCL-1 and BCL-XL and activates BAK-mediated apoptosis in response to protein synthesis inhibition. Genes Dev. 2007;21:929-41.

55. Venturelli OS, Tei M, Bauer S, Chan LJG, Petzold CJ, Arkin AP. Programming mRNA decay to modulate synthetic circuit resource allocation. Nat Commun. 2017:8:15128.

56. Yang J, Jiang W, Yang S. mazF as a counter-selectable marker for unmarked genetic modification of Pichia pastoris. FEMS Yeast Res. 2009;9:600-9.

57. Khetrapal V, Mehershahi K, Rafee S, Chen S, Lim CL, Chen SL. A set of powerful negative selection systems for unmodified Enterobacteriaceae. Nucleic Acids Res. 2015;43:e83.

58. Hughes RA, Ellington AD. Rational design of an orthogonal tryptophanyl nonsense suppressor tRNA. Nucleic Acids Res. 2010;38:6813-30. 
59. Wenthzel AM, Stancek M, Isaksson LA. Growth phase dependent stop codon readthrough and shift of translation reading frame in Escherichia coli. FEBS Lett. 1998;421:237-42.

60. Harris EH, Stern DB, Witman GB. The Chlamydomonas sourcebook. 2nd ed. Oxford: Elsevier Inc.; 2009.

61. Gibson DG, Young L, Chuang RY, Venter JC, Hutchison CA 3rd, Smith HO Enzymatic assembly of DNA molecules up to several hundred kilobases. Nat Methods. 2009;6:343-5.
62. Sambrook J, Russell DW. Molecular cloning: a laboratory manual. Cold Spring Harbor: CSHL Press; 2001.

63. Klein U, De Camp JD, Bogorad L. Two types of chloroplast gene promoters in Chlamydomonas reinhardtii. Proc Natl Acad Sci U S A. 1992:89:3453-7.
Ready to submit your research? Choose BMC and benefit from:

- fast, convenient online submission

- thorough peer review by experienced researchers in your field

- rapid publication on acceptance

- support for research data, including large and complex data types

- gold Open Access which fosters wider collaboration and increased citations

- maximum visibility for your research: over 100M website views per year

At BMC, research is always in progress.

Learn more biomedcentral.com/submissions 